\title{
SpineData - a Danish clinical registry of people with chronic back pain
}

This article was published in the following Dove Press journal:

Clinical Epidemiology

13 August 2015

Number of times this article has been viewed

\author{
Peter Kent ${ }^{1}$ \\ Alice Kongsted ${ }^{1,2}$ \\ Tue Secher Jensen ${ }^{2,3}$ \\ Hanne B Albert ${ }^{4}$ \\ Berit Schiøttz-Christensen ${ }^{3}$ \\ Claus Manniche ${ }^{3}$ \\ 'Department of Sports Science and \\ Clinical Biomechanics, University \\ of Southern Denmark, Odense, \\ Denmark; ${ }^{2}$ Nordic Institute \\ of Chiropractic and Clinical \\ Biomechanics, Odense, Denmark; \\ ${ }^{3}$ Research Department, Spine Centre \\ of Southern Denmark, Hospital \\ Lillebaelt, Institute of Regional Health \\ Research, University of Southern \\ Denmark, Middelfart, Denmark; ${ }^{4}$ The \\ Modic Clinic, Odense, Denmark
}

Background: Large-scale clinical registries are increasingly recognized as important resources for quality assurance and research to inform clinical decision-making and health policy. We established a clinical registry (SpineData) in a conservative care setting where more than 10,000 new cases of spinal pain are assessed each year. This paper describes the SpineData registry, summarizes the characteristics of its clinical population and data, and signals the availability of these data as a resource for collaborative research projects.

Methods: The SpineData registry is an Internet-based system that captures patient data electronically at the point of clinical contact. The setting is the government-funded Medical Department of the Spine Centre of Southern Denmark, Hospital Lillebaelt, where patients receive a multidisciplinary assessment of their chronic spinal pain.

Results: Started in 2011, the database by early 2015 contained information on more than 36,300 baseline episodes of patient care, plus the available 6-month and 12-month follow-up data for these episodes. The baseline questionnaire completion rate has been $93 \% ; 79 \%$ of people were presenting with low back pain as their main complaint, $6 \%$ with mid-back pain, and $15 \%$ with neck pain. Collectively, across the body regions and measurement time points, there are approximately 1,980 patient-related variables in the database across a broad range of biopsychosocial factors. To date, 36 research projects have used data from the SpineData registry, including collaborations with researchers from Denmark, Australia, the United Kingdom, and Brazil.

Conclusion: We described the aims, development, structure, and content of the SpineData registry, and what is known about any attrition bias and cluster effects in the data. For epidemiology research, these data can be linked, at an individual patient level, to the Danish populationbased registries and the national spinal surgery registry. SpineData also has potential for the conduct of cohort multiple randomized controlled trials. Collaborations with other researchers are welcome.

Keywords: back pain, neck pain, thoracic pain, registries, prognosis, outcome measures, quality of care

\section{Background}

Across a range of health conditions, large-scale clinical registries are being compiled to provide a data resource for quality assurance and research to inform clinical decisionmaking and health policy. ${ }^{1-4}$ This epidemiologic activity is especially prevalent in Scandinavia, where there is a long cultural history of widespread data collection, a supportive legislative and ethical framework for registry-based research, and a unique social identification number for each resident (a central person registration number) that allows linkage of information across registries. ${ }^{1,5-7}$ Within the context of the Danish health care system, examples of comprehensive, population-based,
Correspondence: Peter Kent Department of Sports Science and Clinical Biomechanics, University of Southern Denmark, Denmark Campusvej 55, Odense M, 5230, Denmark

Tel +4565501000

Email pkent@health.sdu.dk 
linkable registers include those containing data on the use of primary and secondary health care services, hospital diagnoses, cancers, prescription drugs, education, income, social conditions, geographic location, work participation, and welfare support. ${ }^{1,2}$

Clinical registries create observational cohort data that may be cross-sectional or longitudinal, but the capacity to link those data at an individual patient level with data from other cross-sectional or longitudinal registries provides unique opportunities for scientific inquiry. One example would be research questions addressable using a life-course epidemiology approach.

In the study of back pain, which is the world's leading cause of life years lived with disability, ${ }^{8}$ there has been a recognition by authorities such as the US National Institutes of Health that unique scientific, clinical, and social insights might be gained through the use of large-scale clinical registries. ${ }^{9}$ Similarly, there is increasing interest in the advantages of imbedding randomized clinical trials within the context of registries (cohort multiple randomized controlled trials). ${ }^{10}$

Within the context of a secondary-care hospital department in which approximately 10,000 new patients with spinal pain are seen each year, we established a clinical registry (SpineData). The purposes of the registry were to create a resource for institutional quality assurance and research and to present information in ways that would assist the department's clinicians to make decisions about individual patients. At the time that we established SpineData, a Danish national surgical spine registry (DaneSpine) ${ }^{11}$ was being established, but there was no equivalent national medical spine registry, and that is still the case. Therefore, we established our registry to systematically collect detailed clinical information on an entire cohort of medically managed patients with back pain in secondary care, as a complement to Danish national registries. Currently, all spinal pain patients in this health region who are referred to secondary care are reviewed by the department before being referred elsewhere.

The aims of this paper were to describe the SpineData registry, summarize the characteristics of the clinical population and the content of the data within the registry, and inform the scientific community of the availability of these data as a collaborative resource for research projects.

\section{Methods}

The SpineData registry is an Internet-based, multiuser system that is designed to capture patient data electronically at the point of clinical contact. Patients and health care workers can access the registry 24 hours per day, 7 days per week via web browsers. All information processing is in real time so that summary data are instantly available. The system was developed between mid-2008 and the end of 2010 and was implemented in routine daily patient care in the beginning of 2011.

\section{Setting}

The Medical Department of the Spine Centre of Southern Denmark, Hospital Lillebaelt, is a government-funded facility where patients can be referred from anywhere within a catchment area of 1.2 million people (http://fks. regionsyddanmark.dk/wm202525). Department personnel perform multidisciplinary assessments of patients with spinal pain after referral from general practitioners, chiropractors, and medical specialists in primary care or from other hospital departments. A standardized clinical examination and use of magnetic resource imaging (MRI) are central elements. Patients have a right to be referred if their improvement has not been satisfactory in primary care. The department has branches at multiple hospital sites within the geographical and administrative Region of Southern Denmark.

\section{Inclusion and exclusion criteria}

Any patient attending the Medical Department of the Spine Centre of Southern Denmark for a new episode of care is eligible for inclusion in the SpineData registry. Only patients can exclude themselves by declining participation. Approximately $9 \%$ of patients complete their questionnaires on paper, and because these are back-filled into electronic format when resources are available to do so, at any given point in time some of these data may not yet be available electronically.

\section{Baseline questionnaires Domains and items}

In keeping with the biopsychosocial model of health, information is collected in SpineData across the broad health domains of pain, activity limitation, work participation, psychological factors, physical impairment, and contextual factors. Wherever possible, the choice of questions and questionnaires was based on evidence of their role in the diagnosis, prognosis, or treatment of spinal pain. An example of the reasoning used in that item selection is shown in Supplementary materials.

At the index consultation, both patients and clinicians complete baseline questionnaires. The questions vary across the three spinal regions of principal complaint: neck pain, mid-back pain, and low back pain. The full details of the questions and response options are shown in Table S1. A brief summary of the items collected, using the example of low back pain, is listed here by health domain. ${ }^{12}$ 


\section{Pain domain}

- Patient-reported questions: main pain chart (current pain) and other pain chart (any additional locations of pain during the previous 2 weeks) (46 body areas each), onset date of pain, any previous low back pain or sciatica episodes, low back pain intensity (current, typical, and worst in last 14 days), leg pain intensity (current, typical, and worst in last 14 days), numbers of days per week with pain, buttock pain, pain shifting from side to side, cause of or reason for onset, morning stiffness, diurnal variation, movement-related pain, activity-related pain, effect of physical rest on pain, pain easily aggravated by movement, and pain that takes a long time to settle.

\section{Activity limitation domain}

- Patient-reported questions: the 23-item Roland-Morris Disability Questionnaire.

\section{Participation domain}

- Patient-reported questions: type of employment, whether on sick leave due to back pain any time in the last 3 months and for how long, on reduced work hours due to back pain, expectation of being able to sit or stand long enough for normal work in 6 weeks' time, expectation of working in 6 months, physically strenuous work, monotonous work, and work satisfaction.

\section{Psychological domain (mental functions)}

- Patient-reported questions: anxiety, depression, pain catastrophization, fear of movement, self-rated risk of pain persistence, social isolation, and the STarT Back Tool classifications. ${ }^{13}$

\section{Physical impairment domain (neuromusculoskeletal and movement-related function)}

- Patient-reported questions: lower limb weakness, altered groin or rectal sensation.

- Clinician-reported questions: lower limb muscle strength, atrophy, sensation, deep tendon reflexes, Straight Leg Raise Test, ${ }^{14}$ Prone Knee-Bend Test, ${ }^{15}$ spinal springing tests ${ }^{16}$ sacroiliac joint tests (Gaenslen's Test, ligament tenderness, Posterior Pelvic Pain Provocation Test), ${ }^{17}$ elbow hyperextension as an indication of systemic hypermobility, aberrant spinal movement, pain on active movement in cardinal directions, directional preference (mechanical diagnosis and therapy), and paraspinal tenderness.

\section{Contextual (environmental and personal)}

\section{factors domain}

- Patient-reported questions: height, weight, previous back surgery, prolonged corticosteroid use, exposure to prolonged mechanical vibration, handedness, level of recreational physical activity, allergies, cigarette use, alcohol consumption, making an insurance claim due to back pain, applying for a pension due to back pain, workmate relations, serious lung disease, heart disease, cancer, and use of analgesics.

- Generated from central person registration number: age and sex.

- Clinician-reported questions: medication use (acetylsalicylic acid, codeine, morphine, non-steroidal antiinflammatory drugs, acetaminophen, tramadol, other analgesics).

\section{Follow-up questionnaires}

All patients are invited to complete two follow-up questionnaires, which contain approximately half the questions in the baseline patient questionnaires. Prior to January 1, 2012, the first follow-up questionnaire occurred 3 months after the date of the initial consultation. After that date, the first follow-up was collected at 6 months, as this was believed to be a more suitable outcome time point in the clinical course of people with chronic pain. The second follow-up questionnaire has always occurred at 12 months.

\section{Languages and voice recordings}

To accommodate patients within the population seen at the Spine Centre who are not native Danish speakers, the patient questionnaires and all webpage instructions have been translated from English into Danish, German, Arabic, Persian, Serbian, Somali, Tamil, Turkish, and Vietnamese (translations are freely available on request from the Spine Centre). Where validated questionnaires were available in the target languages, these were used Otherwise, the questionnaires were forward-translated and back-translated by professional translators, but due to a lack of resources, they were not further validated. In this way, patients can complete the questionnaires in the language of their choice, and clinicians can see the results in the language of their choice (Danish). In practice, only 1.8\% of the questionnaires are being completed in languages other than Danish.

To accommodate the $10 \%$ or more patients believed to have some level of functional illiteracy, explanations of all self-reported questions were voice-recorded in English and 
Danish and are made available to patients via an on-screen symbol.

\section{Reports}

Summary reports are generated in real-time for clinicians and administrative staff, either on an individual patient level or for groups of patients. For example, before seeing a patient for the initial consultation, clinicians can see summary reports from that patient's self-reported baseline questionnaire and can access summaries of baseline data and outcomes of any previous episodes for that patient. Collectively, across the body regions and measurement time points, there are approximately 1,980 patient-related variables in the database.

\section{Ethics}

Ethics approval for the collection and use of these data for quality assurance and research purposes was approved by the Scientific Committee of the Region of Southern Denmark (project ID S-200112000-29). The database is registered with the Danish Data Protection Agency (2008-58-0035).

All patients are invited to give two types of written informed consent. The first is for their individual patient data to be used for quality assurance and research purposes, including in publications of anonymized group-level data. The second is for the Spine Centre to contact them requesting completion of follow-up questionnaires.

\section{Technology}

The SpineData registry is administered using a custom-built software layer (Utility Creative, Melbourne, Australia) within an open-source software stack (MSQL, Java, Apache Tomcat, JQuery) running on a server managed by the governmental administration of the Region of Southern Denmark. The software allows the self-management of questions, questionnaire design, and data management by the Clinical Department. It also allows the integration of multiple simultaneous research projects into the department's usual workflow, by dynamically changing the questionnaire experience depending on project participation. The use of conditional logic, where specific responses to questions determine which subsequent questions will be displayed, results in a reduced length of questionnaires for individual patients. Questionnaires include graphical methods of capturing information that are translated into numeric data in real-time. Examples of questionnaire webpages are shown in Figures 1 and 2.

The questionnaire functionality was also extended in an imaging module to allow the quantitative coding of MRI findings, including allowing for multiple simultaneous research projects (approximately 2,230 MRI variables collectively across all coding models). As part of this functionality, the registry interfaces with the local radiology imaging system (radiology information system/picture archiving and communications system). The registry is also linked to the Danish Central Persons Registry to enable access to up-todate information about patient contact details.

All patients can complete registry questionnaires from home using computers, tablets, or smart phones, and the user interface is adaptive to the type of device that they are using. They can also complete the questionnaires at the Spine Centre using in-house touch screen computers or tablets. Spine Centre personnel also access the registry using in-house computers.

The registry has been built to meet all Danish health data security standards for data access, storage, backup, and the tracking of who accesses, inputs, or modifies data. This is achieved via a layered approach of login requirements, passwords, firewalls, data functionality limited to discrete IP addresses, logging of access, and database architecture designed to resist denial of service attacks.

The SpineData software is now being used by other hospital departments for other health conditions.

\section{Funding}

The registry was developed with the support of a mixture of private and public funding. Private research funding was sourced from grants from Industriens Arbejdsskadeforsikring and Trygfonden. In addition, project grants from the Danish Foundation for Chiropractic Research and Post-graduate Education facilitated researchers to collect data using the registry and thereby build the capacity of the registry. The remaining development costs and ongoing maintenance costs have been met by the Medical Department of the Spine Centre.

\section{Results}

\section{Patient population characteristics}

At the beginning of 2015, the database contained information on more than 36,300 baseline episodes of patient care (31,746 unique patients) plus the available follow-up data for these episodes. The baseline questionnaire completion rate has been $93.4 \%$ of all registered patients, with approximately $79 \%$ of people in the database having presented with low back pain as their main complaint, $6 \%$ with mid-back pain, and $15 \%$ with neck pain. The main clinical characteristics of this chronic-pain cohort at baseline and at 12 months are 


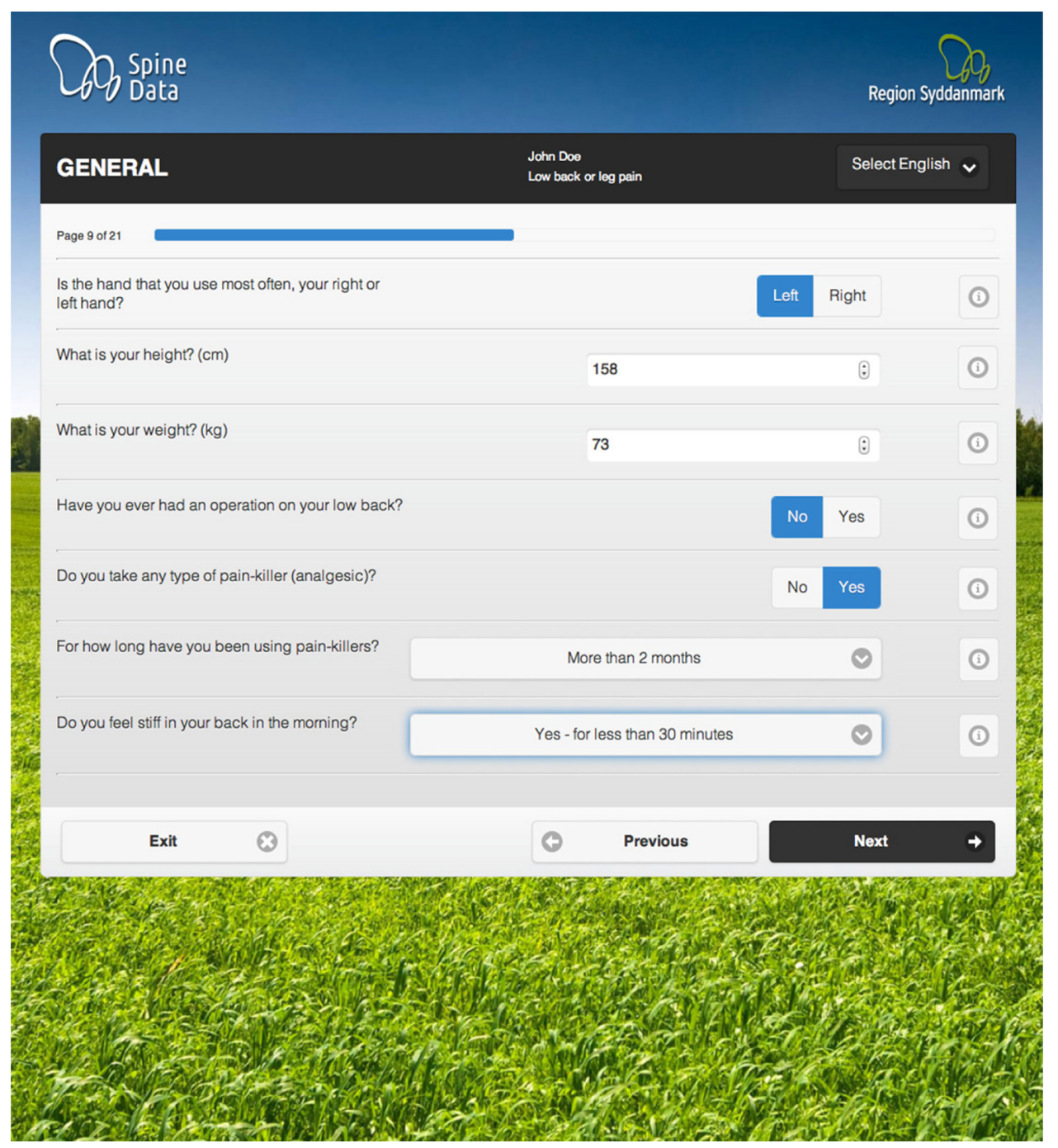

Figure I Example patient questionnaire webpage.

summarized in Table 1. Figure 3 is a flowchart of participation in the registry from January 1, 2011 to July 17, 2014. Most patients had chronic pain, with $83 \%$ having an episode duration of 3 months or more.

The current consent rate for use of personal data for quality assurance and research use is $97.7 \%$, and the consent rate for being sent follow-up questionnaires is $83.5 \%$. The current completion rate of follow-up questionnaires is $50.8 \%$ of consenters ( $40.4 \%$ of the entire cohort) at 6 months and $35.7 \%$ of consenters $(28.4 \%$ of the entire cohort) at 12 months.

As participants are patients seeking care in a medical department, their participation in the registry is incidental to the main purpose of their attendance, and the personal health information that they volunteer will inform their care. Therefore, we believe that we cannot compel patients to answer every question in the questionnaires and, as a result, almost all questions are nonmandatory. The only compulsory questions are those required for the department to meet its obligatory quality assurance requirements. Despite this, the amount of missing data on individual questions is relatively low. For example, the median amount of missing data in the core questions asked of all patients in the low back pain questionnaire is $7 \%$ (interquartile range [IQR] $6 \%$ to $8 \%$, full range $0 \%$ to $13 \%$ ).

\section{Follow-up attrition bias}

To test for attrition bias, in the cohort of patients whose primary complaint was low back pain, we randomly selected 300 people who completed the follow-up questionnaires and, from the same time period, randomly selected 300 people who did not. Using STATA 12 (StataCorp LP, College Station, TX, USA), we compared these groups on their baseline characteristics of age, sex, episode duration, 


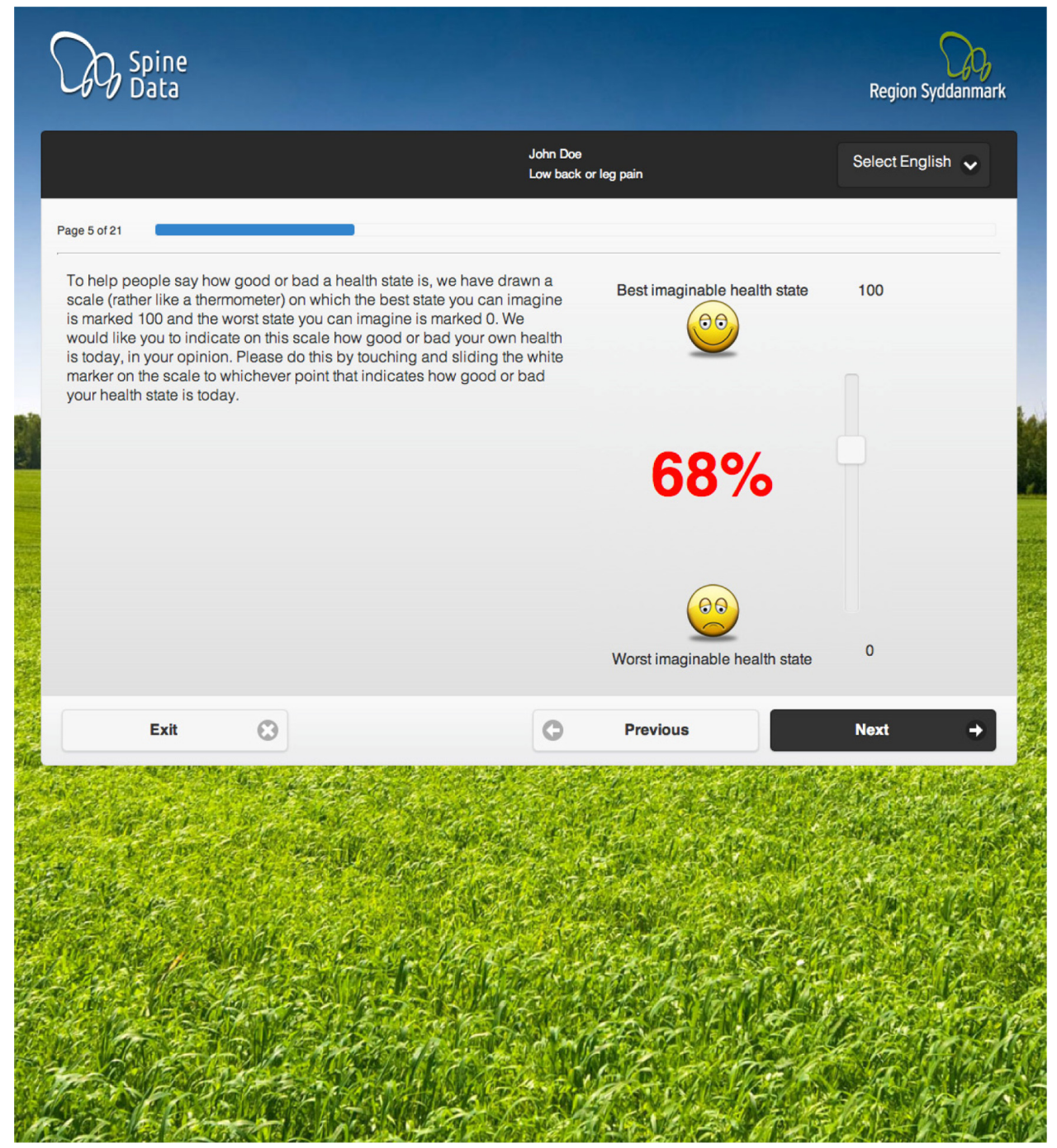

Figure 2 Example patient questionnaire graphical interface.

back pain intensity, leg pain intensity, pain-related activity limitation, fear of movement, depression, and self-perceived general health.

We also contacted a consecutive sample of people who did not spontaneously complete follow-up questionnaires and encouraged them to do so, until we had 200 6-month questionnaires and 200 12-month questionnaires. We then compared these with 300 questionnaires from randomly selected spontaneous completers from each time point on the outcomes of low back pain intensity, leg pain intensity, pain-related activity limitation, and change from baseline in each of those outcome measures.

Those people who did not complete the 6-month questionnaire were significantly younger at baseline age (noncompleters $47.8 \pm 16.1$ years versus completers $55.9 \pm 15.5$ years; $P<0.01)$ and had a higher prevalence of depression (noncompleters $22.8 \%$ [95\% CI 17.8\%-27.8\%] versus completers $16.1 \%$ [95\% CI $11.8 \%-20.3 \%$ ]; $P=0.04$ ) but they did not significantly differ on any of the outcomes at 6 months (see Table 2 for further details), even when controlling for baseline differences in age and depression (linear regression $P=0.13-0.80$ ). Therefore, there does not appear to be any attrition bias in the 6-month questionnaire data on the commonly used outcomes, but there is an attrition bias on the baseline characteristics of age and depression.

Those people who did not complete the 12-month questionnaire were significantly younger at baseline (noncompleters $47.8 \pm 15.8$ years versus completers $56.7 \pm 14.7$ years; $P<0.01)$ and had a higher baseline fear of movement (noncompleters $4.3 \pm 3.3$ versus completers $3.7 \pm 3.1 ; P=0.01$ ). In addition, they also had higher low back pain intensity scores at 12 months (noncompleters $5.2 \pm 2.6$ versus completers $4.5 \pm 2.7 ; P<0.01$ ) and low back 
Table I Characteristics of clinical population at baseline and at the I2-month follow-up

\begin{tabular}{|c|c|c|}
\hline Clinical characteristic & Baseline & 12 months \\
\hline Age, years; mean (SD, full range) & $49.8(15.2 ; 15.2-99.2)$ & \\
\hline Sex (female) & $55.6 \%$ & \\
\hline Episode duration (months); median (IQR; full range) & II.3 (4.I-37.6; 0.6-365.4) & \\
\hline Any pain-related sick leave during previous 3 months (in working population) & $50.1 \%$ & $27.5 \%$ \\
\hline Fear of movement (0-10); mean (SD)* & $7.9(6.1)$ & $3.3(3.1)$ \\
\hline Depression (yes) & $19.7 \%$ & $16.8 \%$ \\
\hline Anxiety $(0-10) ;$ mean $(S D)^{*}$ & $3.5(3.1)$ & $2.7(3.0)$ \\
\hline Pain catastrophization $(0-10)$; mean $(S D)^{*}$ & $4.1(3.0)$ & $3.5(2.9)$ \\
\hline Self-perceived general health, EuroQoL health thermometer (0-100); mean (SD)* & $49.3(23.9)$ & $61.3(24.1)$ \\
\hline \multicolumn{3}{|l|}{ Spinal area of main complaint } \\
\hline Neck pain & $15.2 \%$ & \\
\hline Mid-back pain & $5.8 \%$ & \\
\hline Low back pain & $79.0 \%$ & \\
\hline \multicolumn{3}{|l|}{ Neck pain, Quebec Task Force classification } \\
\hline Local neck pain & $10.1 \%$ & \\
\hline Neck pain and arm pain above elbow & $16.1 \%$ & \\
\hline Neck pain and arm pain below elbow & $24.4 \%$ & \\
\hline Neck pain and nerve root irritation & $46.4 \%$ & \\
\hline Neck pain intensity, NRS (0-10); mean (SD) & $6.0(2.4)$ & $4.4(2.8)$ \\
\hline Arm pain intensity, NRS $(0-10)$; mean $(S D)$ & $4.8(3.0)$ & $3.5(3.0)$ \\
\hline Neck activity limitation, Neck Disability Index (0-100); mean (SD) & $41.7(17.8)$ & $33.1(20.1)$ \\
\hline Satisfied or very satisfied with care ${ }^{\#}$ & & $69 \%$ \\
\hline Mid-back pain intensity, NRS (0-10); mean (SD) & $6.2(2.1)$ & $5.1(2.6)$ \\
\hline Chest pain intensity, NRS (0-10); mean (SD) & $1.9(2.7)$ & $2.0(2.6)$ \\
\hline Mid-back activity limitation, Modified Neck Disability Index (0-100); mean (SD) & $40.7(17.0)$ & $31.6(18.7)$ \\
\hline Satisfied or very satisfied with care ${ }^{\#}$ & & $64 \%$ \\
\hline \multicolumn{3}{|l|}{ Low back pain, Quebec Task Force classification } \\
\hline Local low back pain & $18.5 \%$ & \\
\hline Low back pain and leg pain above elbow & $11.6 \%$ & \\
\hline Low back pain and leg pain below elbow & $22.4 \%$ & \\
\hline Low back pain and nerve root irritation & $47.5 \%$ & \\
\hline Low back pain intensity, NRS (0-10); mean (SD) & $5.9(2.4)$ & $4.5(2.7)$ \\
\hline Leg pain intensity, NRS $(0-10)$; mean (SD) & $4.7(2.9)$ & $3.3(2.9)$ \\
\hline Low back activity limitation, RMDQ (0-100); mean (SD) & $62.8(23.4)$ & $47.4(29.4)$ \\
\hline Satisfied or very satisfied with care & & $69 \%$ \\
\hline
\end{tabular}

Notes: Lower scores are better on all questionnaire scales except for the EuroQoL health thermometer, where higher scores are better. *Measured using psychosocial screening questions and thresholds validated in this setting; ${ }^{1 \#}$ measured on a seven-point Likert Scale (very satisfied, satisfied, a little satisfied, neither satisfied nor dissatisfied, a little dissatisfied, dissatisfied, very dissatisfied).

Abbreviations: IQR, interquartile range; NRS, numeric rating scale; RMDQ, Roland-Morris Disability Questionnaire (23-item version); SD, standard deviation.

pain intensity change scores from baseline to 12 months (non-completers $0.8 \pm 2.6$ versus completers $1.3 \pm 2.7$; $P=0.05)$. Therefore, in the low back pain 12-month questionnaire data, there is an attrition bias on one of the commonly used outcomes (back pain intensity) and also on the baseline characteristics of age and fear of movement (see Table 3 for further details).

\section{Variability in outcomes due to different clinicians and clinical teams}

To gain an indication of the magnitude of any effects due to differences between individual clinicians and clinical teams (cluster effects) that might affect estimates based on the data, we conducted longitudinal mixed modeling of the two most-common outcomes used: pain intensity and pain-related activity limitation, using the low back pain data as an example (arbitrarily a sample of $n=2,351$ consecutive patients). Using baseline, 3-month, and 12-month outcomes data, we used a likelihood ratio test to compare the results of simple (two-level: patients and timepoints) longitudinal models with results from multilevel models that included levels for the 89 individual clinicians and seven teams of clinicians who contributed data.

There were no statistically significant differences due to clinician or clinical team effects on either outcome $(P=0.18-1.00)$, and intraclass correlations were negligible $(0.000-0.002)$. The magnitude of these cluster effects may vary, however, depending on which independent and dependent variables are used, and these results should therefore be interpreted as only providing a general indication. 


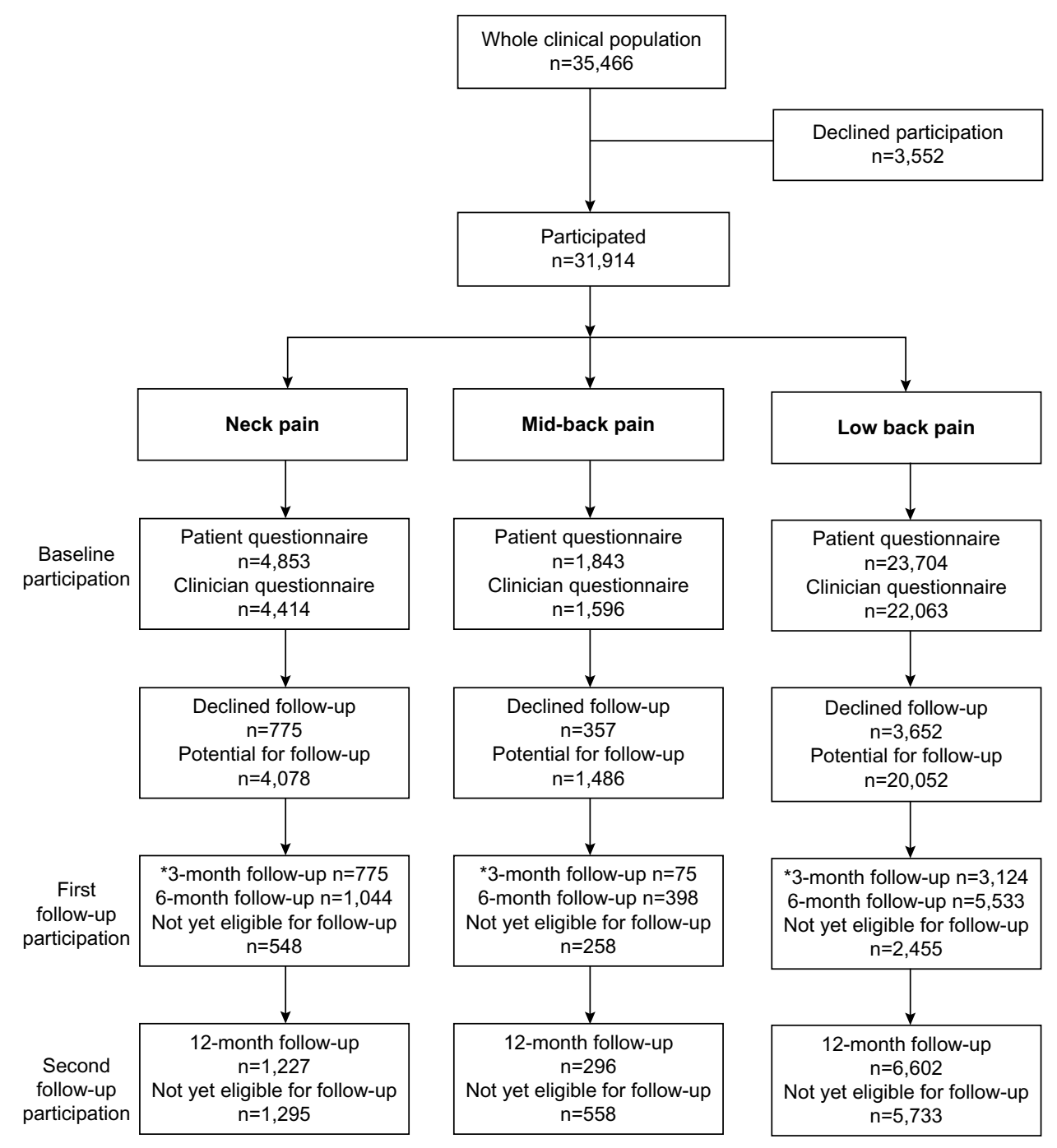

Figure 3 Flowchart of registry participation between January I, 20II and July I7, 2014.

Note: *Before January I, 20I2, the first follow-up questionnaire occurred 3 months after the date of the initial consultation.

\section{Discussion}

We have described the aims, development, structure, and content of the SpineData registry. We have also reported what is known about attrition bias and cluster effects in the data. Some of the potential of the data has been described, including the potential for data linkage with other Danish population-based registries.

\section{Research projects}

To date, 36 research projects have used data from the SpineData registry, resulting in a number of publications ${ }^{18-27}$ and reports, with more in press or under preparation. These projects have included collaborations between researchers from Denmark, Australia, the United Kingdom, and Brazil. An example of a research project that was imbedded in SpineData is a spondyloarthropathy study of 1,037 patients aged 18-40 years referred with persistent low back pain and no previous suspicion of spondyloarthropathy. ${ }^{18,19}$ In addition to the usual SpineData questions, additional data collection included quantified MRI findings from the whole spine and the sacroiliac joints, blood samples, and spondyloarthropathy-related questionnaires. The aims of the study were 1) to estimate the baseline prevalence of MRI findings and clinical characteristics previously associated with spondyloarthropathy, 2) investigate the associations between MRI findings and baseline clinical characteristics suggestive of spondyloarthropathy, 3) describe the development of spondyloarthritis-related MRI findings at the 3-year follow-up, and 4) examine which baseline MRI findings and clinical findings predict the development of severe spondyloarthritis at the 3 -year follow-up. This project was a data linkage collaboration between specialist departments across three hospitals: King Christian 10th Hospital for 
Table 2 Comparison of completers and noncompleters of 6-month low back pain questionnaire

\begin{tabular}{|c|c|c|c|}
\hline Baseline & $\begin{array}{l}\text { Noncompleters } \\
n=200\end{array}$ & $\begin{array}{l}\text { Completers } \\
n=300\end{array}$ & $P$-value \\
\hline \multicolumn{4}{|l|}{ Characteristics } \\
\hline Age (years); mean (SD) & $47.8(16.1)$ & $55.9(\mid 5.5)$ & $<0.01$ \\
\hline Sex (female); proportion (IQR) & $56.9 \%(51.2 \%-62.5 \%)$ & $50.0 \%(44.3 \%-55.7 \%)$ & 0.09 \\
\hline Episode duration (months); median (IQR) & $12.2(4.4-49.9)$ & $9.7(3.8-36.7)$ & 0.08 \\
\hline Low back pain intensity, NRS (0-10); mean (SD) & $6.00(2.4 I)$ & $5.79(2.47)$ & 0.14 \\
\hline Leg pain intensity, NRS (0-I0); mean (SD) & $4.60(3.14)$ & $4.56(2.90)$ & 0.89 \\
\hline Activity limitation, RMDQ (0-100); mean (SD) & $62.03(25.78)$ & $61.76(22.79)$ & 0.90 \\
\hline Fear of movement $(0-10)$; mean (SD)* & $3.91(3.30)$ & $3.83(3.30)$ & 0.76 \\
\hline Depression; proportion (IQR)* & $22.8 \%(17.8 \%-27.8 \%)$ & $16.1 \%(11.8 \%-20.3 \%)$ & 0.04 \\
\hline Self-perceived general health $(0-100)$; mean $(S D)^{\#}$ & $49.53(23.90)$ & $50.4(24.06)$ & 0.67 \\
\hline \multirow[t]{2}{*}{ 6-month outcomes } & Prompted-completers & Completers & \\
\hline & $\mathrm{n}=\mathbf{2 0 0}$ & $n=300$ & \\
\hline Low back pain intensity, NRS (0-10); mean (SD) & $4.69(2.60)$ & $4.25(2.57)$ & 0.07 \\
\hline Leg pain intensity, NRS (0-10); mean (SD) & $3.06(2.62)$ & $3.17(2.80)$ & 0.65 \\
\hline Activity limitation (0-I00); mean (SD) & $48.0(28.6)$ & $44.5(28.4)$ & 0.20 \\
\hline Low back pain change score (-10 to 10$)$; mean (SD) & $1.49(2.53)$ & $\mathrm{I} .23(2.5 \mathrm{I})$ & 0.28 \\
\hline Leg pain intensity change score ( -10 to 10$)$; mean (SD) & $1.84(3.35)$ & $1.57(3.26)$ & 0.40 \\
\hline Activity limitation change score (-10 to 10$)$; mean (SD) & I6.4I (23.68) & I $6.07(27.36)$ & 0.90 \\
\hline
\end{tabular}

Notes: Compared with Student's $t$-tests, Mann-Whitney $U$ tests, or tests of proportions, depending on the data distributions. *Measured using one-item or two-item psychosocial screening questions and thresholds validated in this setting for each construct; ${ }^{17}{ }^{*}$ measured using the EuroQoL health thermometer.

Abbreviations: IQR, interquartile range; NRS, numeric rating scale; RMDQ, Roland-Morris Disability Questionnaire (23-item version); SD, standard deviation.

Rheumatic Diseases at Graasten (biochemical data), Aarhus University Hospital (radiologic data), and Hospital Lillebaelt (medical data).

This registry, and the clinical setting in which it occurs, are also a potential resource and location in which to conduct pragmatic clinical trials using the "cohort multiple randomized controlled trial" design. ${ }^{10}$ In this design, also referred to as a "cohort design randomized controlled trial," patients for the intervention arm are randomly recruited from an existing cohort study and their outcomes compared with those of the existing cohort. It has a number of advantages, including the cohort providing a ready source for recruitment,

Table 3 Comparison of completers and noncompleters of 12-month low back pain questionnaire

\begin{tabular}{|c|c|c|c|}
\hline Baseline & $\begin{array}{l}\text { Noncompleters } \\
n=300\end{array}$ & $\begin{array}{l}\text { Completers } \\
n=300\end{array}$ & $P$-value \\
\hline \multicolumn{4}{|l|}{ Characteristics } \\
\hline Age (years); mean (SD) & $48.7(15.8)$ & $56.7(14.7)$ & $<0.01$ \\
\hline Sex (female); proportion (IQR) & $53.5 \%(47.9 \%-59.2 \%)$ & $54.3 \%(48.7 \%-60.0 \%)$ & 0.84 \\
\hline Episode duration (months); median (IQR) & II.5 (4.I-38.4) & $9.8(4.0-184.2)$ & 0.69 \\
\hline Low back pain intensity, NRS (0-I0); mean (SD) & $5.68(2.31)$ & $5.53(2.38)$ & 0.43 \\
\hline Leg pain intensity, NRS (0-10); mean (SD) & $4.39(3.05)$ & $4.78(2.68)$ & 0.10 \\
\hline Activity limitation, RMDQ (0-100); mean (SD) & $62.5(24.0)$ & $60.9(22.17)$ & $0.4 I$ \\
\hline Fear of movement $(0-10) ;$ mean $(S D)^{*}$ & $4.32(3.29)$ & $3.66(3.08)$ & 0.01 \\
\hline Depression; proportion (IQR)* & $19.3 \%(14.7 \%-24.0 \%)$ & $18.7 \%(14.1 \%-23.2 \%)$ & 0.84 \\
\hline Self-perceived general health $(0-100)$; mean $(S D)^{\#}$ & $49.7(22.8)$ & $50.0(23.5)$ & 0.89 \\
\hline \multirow[t]{2}{*}{ I2-month outcomes } & Prompted-completers & Completers & \\
\hline & $\mathrm{n}=\mathbf{2 0 2}$ & $\mathrm{n}=\mathbf{3 0 0}$ & \\
\hline Low back pain intensity, NRS (0-I0); mean (SD) & $5.19(2.55)$ & $4.48(2.73)$ & $<0.01$ \\
\hline Leg pain intensity, NRS (0-10); mean (SD) & $3.05(2.99)$ & $3.12(2.92)$ & 0.80 \\
\hline Activity limitation (0-100); mean (SD) & $50.3(28.3)$ & $50.0(29.8)$ & 0.40 \\
\hline Low back pain change score (-10 to 10$)$; mean (SD) & $0.80(2.55)$ & $\mathrm{I} .28(2.7 \mathrm{I})$ & 0.05 \\
\hline Leg pain intensity change score $(-10$ to 10$)$; mean (SD) & $1.53(3.32)$ & $1.57(3.18)$ & 0.88 \\
\hline Activity limitation change score (-10 to 10$)$; mean (SD) & I I.63 (27.27) & 12.01 (26.93) & 0.88 \\
\hline
\end{tabular}

Notes: Compared with Student's t-tests, Mann-Whitney $U$ tests or tests of proportions, depending on the data distributions. $*$ Measured using one-item or two-item psychosocial screening questions and thresholds validated in this setting for each construct; ${ }^{17}$ measured using the EuroQoL health thermometer.

Abbreviations: IQR, interquartile range; NRS, numeric rating scale; RMDQ, Roland-Morris Disability Questionnaire (23-item version); SD, standard deviation. 
increased efficiency, continuous information about the outcomes of treatment as usual, and better comparability between trials undertaken using the same cohort. Such trials have been shown to be feasible in low back pain settings. ${ }^{28}$

In parallel with SpineData, the Surgical Department at the Spine Centre of Southern Denmark also manages the Danish national surgical spine registry (DaneSpine) ${ }^{11}$, which contains detailed information on surgical procedures and postoperative outcomes at up to 5-year follow-up. This allows the potential linkage of data for SpineData patients who do and do not go to surgery.

Researchers who are interested in accessing data from SpineData and participating in collaborative projects can contact the department (Rygcenter Syddanmark in Danish). There is a Data Access Committee and a transparent ethical/ procedural framework, and English/Danish language data access application forms are available.

\section{Follow-up rate}

The follow-up completion rate has varied over time and currently is $51 \%$ of consenters at 6 months and $36 \%$ at 12 months. We instituted a number of measures that were designed to improve participation, including: 1) allowing patients to nominate their preference for the contact method (postal letter, SMS text message, email) by which we would advise them that it was time to complete the questionnaire; 2) allowing patients to nominate their preference for being posted a paper questionnaire or completing the questionnaire electronically; 3) providing automated reminders (SMS text message or email) to those who had not completed the questionnaire; and 4) building an adaptive software interface so that questionnaires could be completed on mobile phones, computer tablets, or desktop computers. Despite this, the completion rate remains a weakness of the registry. This may be a reflection of the registry not being a discrete research project where only people who agree to full participation are included. In contrast, it is a near-complete cohort of all people referred for care to the main regional spine center, and potential participants have no obligation to participate in any way. Other spinal registries report varying follow-up rates, from Spine Tango, which has a $16 \%$ follow-up rate at $3-6$ months, ${ }^{29}$ to DaneSpine, which has achieved a 12 -month follow-up rate of $84 \%$ by using the phone to contact every person not completing a questionnaire within the nominated time period. ${ }^{11}$

\section{Strengths and weaknesses}

The SpineData registry has a number of strengths. It is a consecutive cohort of all patients referred to a regional secondary care center, which improves it generalizability.
It is a large, comprehensive dataset covering all three spinal areas (neck, mid-back, low back). The recording of each patient's central person registration number facilitates data linkage with other Danish registries. The size of the dataset improves the capacity to study rare events.

This registry also has a number of weaknesses. Firstly, less than half the patients complete follow-up questionnaires and although we could only find evidence of attrition bias in one outcome at one time point, there were differences in the baseline characteristics of responders and nonresponders.

In addition, although we have conducted periodic staff training that details the reasons underpinning the clinical examination procedures used by clinicians, the quality assurance of data collected by clinicians in daily routine care is not strictly standardized and may be different from that collected in a discrete research project. ${ }^{30}$ For example, that staff training included reaching a consensus about which methods for performing a neurological examination would become the departmental standard and how the results would be quantified. For instance, the sequence of physical components of the Straight Leg Raise Test that would be used, given the diverse ways that this test has been described, and what specifically would indicate a positive test result. However, in contrast to a research project, individual clinicians in routine care are not monitored for adherence to that department standard.

Similarly, routine data collection is different from collecting data to answer a specific research question, as it involves a trade-off between using detailed and validated questionnaires and being able to cover a number of potentially important aspects of spinal pain. For example, in the SpineData registry, we use one-item and two-item screening questions to assess the psychosocial constructs of depression, anxiety, fear of movement, pain catastrophization, and social isolation. Although these screening questions have known concurrent validity in this clinical setting, ${ }^{21}$ relative to full reference standard questionnaires, their relative responsiveness is unknown.

\section{Conclusion}

The SpineData registry contains data from a large and comprehensive observational cohort of consecutive patients attending a nonsurgical hospital department for the multidisciplinary assessment of chronic spinal pain. It contains detailed baseline and outcomes data on a broad range of biopsychosocial factors. For epidemiology research, these data can be linked, at an individual patient level, to the Danish population-based registries and the national spinal surgery registry. SpineData is also a potential setting in which to conduct cohort multiple randomized controlled trials. Collaborations with other researchers are welcome. 


\section{Acknowledgments}

PK is partially funded, and TSJ and AK are fully funded, by the Danish Fund for Chiropractic Research and Post-graduate Education. No funding source played any role in the scientific conduct of the study.

\section{Author contributions}

All authors made substantial contributions to the data generation and analysis, drafting or critical revision of the manuscript and approval for the final version to be published.

\section{Disclosure}

PK, TSJ, and the Spine Centre of Southern Denmark hold partial intellectual property rights to the software developed for the registry, but no individual has gained financially from this beyond their normal salary paid to work on the project. The authors declare that they have no other financial competing interests. The authors report no other conflicts of interest in this work.

\section{References}

1. Thygesen LC, Daasnes C, Thaulow I, Brønnum-Hansen H. Introduction to Danish (nationwide) registers on health and social issues: structure, access, legislation, and archiving. Scand J Public Health. 2011; 39(7 Suppl):12-16.

2. Thygesen LC, Ersbøll AK. Danish population-based registers for public health and health-related welfare research: introduction to the supplement. Scand J Public Health. 2011;39(7 Suppl):8-10.

3. National Institutes of Health. NIH clinical research trials and you: list of registries [webpage on the Internet]. Bethesda, MD: National Institutes of Health; 2015 [updated April 23, 2015]. Available from: http://www.nih. gov/health/clinicaltrials/registries.htm. Accessed January 23, 2015.

4. Monash University. Registries at the School of Public Health and Preventive Medicine [webpage on the Internet]. Victoria, Australia: Monash University; 2015 [updated February 3, 2014]. Available from: http://www.med.monash.edu.au/sphpm/depts-centres-units/registries/ sphpm-registries.html. Accessed May 17, 2015.

5. Frank L. Epidemiology. The epidemiologist's dream: Denmark. Science. 2003;301(5630):163.

6. Andersen CL, Siersma VD, Karlslund W, et al. The Copenhagen Primary Care Differential Count (CopDiff) database. Clin Epidemiol. 2014;6:199-211.

7. Sortsø C, Thygesen LC, Brønnum-Hansen H. Database on Danish population-based registers for public health and welfare research. Scand J Public Health. 2011;39(7 Suppl):17-19.

8. Hoy D, March L, Brooks P, et al. The global burden of low back pain: estimates from the Global Burden of Disease 2010 study. Ann Rheum Dis. 2014;73(6):968-974.

9. Olsen LA, Grossman C, McGinnis JM, editors. Institute of Medicine (US) Roundtable on Value and Science-Driven Health Care. Appendix C. Comparative effectiveness research priorities: IOM recommendations (2009). Learning What Works: Infrastructure Required for Comparative Effectiveness Research: Workshop Summary; 2008 Jul 30-31; Washington, DC. Washington, DC: National Academies Press (US); 2011.

10. Relton C, Torgerson D, O'Cathain A, Nicholl J. Rethinking pragmatic randomised controlled trials: introducing the "cohort multiple randomised controlled trial" design. BMJ. 2010;340:c1066.

11. Simony A, Hansen KH, Ernst C, Andersen MO. [Implementation of the Danish national database Danespine for spinal surgery]. Ugeskr Laeger. 2014;176(2A):V01130019. Danish.
12. World Health Organization. International Classification of Functioning, Disability and Health: ICF. Geneva, Switzerland: World Health Organization; 2001.

13. Hill JC, Dunn KM, Lewis M, Mullis R, Main CJ, Foster NE, Hay EM: A primary care back pain screening tool: identifying patient subgroups for initial treatment. Arthritis Rheum. 2008;59(5):632-641.

14. Devillé WL, van der Windt DA, Dzaferagić A, Bezemer PD, Bouter LM. The test of Lasègue: systematic review of the accuracy in diagnosing herniated discs. Spine (Phila Pa 1976). 2000;25(9):1140-1147.

15. Suri P, Rainville J, Katz JN, Jouve C, Hartigan C, Limke J, Pena E, Li L, Swaim B, Hunter DJ. The accuracy of the physical examination for the diagnosis of midlumbar and low lumbar nerve root impingement. Spine (Phila Pa 1976). 2011;36(1):63-73.

16. Schneider M, Erhard R, Brach J, Tellin W, Imbarlina F, Delitto A. Spinal palpation for lumbar segmental mobility and pain provocation: an interexaminer reliability study. J Manipulative Physiol Ther. 2008;31(6):465-473.

17. Vleeming A, Albert HB, Ostgaard HC, Sturesson B, Stuge B. European guidelines for the diagnosis and treatment of pelvic girdle pain. Eur Spine J. 2008;17(6):794-819.

18. Arnbak B. The Use of MRI in the Early Diagnosis of Spondyloarthritis: A Cross-Sectional Cohort Study of Secondary Care Patients with Persistent Low Back Pain [doctoral thesis]. Odense, Denmark: Institute of Regional Health Research, University of Southern Denmark; 2014.

19. Arnbak B, Jensen TS, Manniche C, Zejden A, Egund N, Jurik AG. Spondyloarthritis-related and degenerative MRI changes in the axial skeleton - an inter- and intra-observer agreement study. BMC Musculoskelet Disord. 2013;14:274.

20. Johansen JV, Manniche C, Kjaer P. Vitamin D levels appear to be normal in Danish patients attending secondary care for low back pain and a weak positive correlation between serum level Vitamin D and Modic changes was demonstrated: a cross-sectional cohort study of consecutive patients with non-specific low back pain. BMC Musculoskelet Disord. 2013;14:78.

21. Kent P, Mirkhil S, Keating J, Buchbinder R, Manniche C, Albert HB. The concurrent validity of brief screening questions for anxiety, depression, social isolation, catastrophization, and fear of movement in people with low back pain. Clin J Pain. 2014;30(6):479-489.

22. Kløjgaard ME, Manniche C, Pedersen LB, Bech M, Søgaard R. Patient preferences for treatment of low back pain-a discrete choice experiment. Value Health. 2014;17(4):390-396.

23. Kongsted A, Kent P, Albert H, Jensen TS, Manniche C. Patients with low back pain differ from those who also have leg pain or signs of nerve root involvement - a cross-sectional study. BMC Musculoskelet Disord. 2012; $13: 236$.

24. Kongsted A, Kent P, Jensen TS, Albert H, Manniche C. Prognostic implications of the Quebec Task Force classification of back-related leg pain: an analysis of longitudinal routine clinical data. BMC Musculoskelet Disord. 2013;14:171.

25. Morsø L, Kent P, Manniche C, Albert HB. The predictive ability of the STarT Back Screening Tool in a Danish secondary care setting. Eur Spine J. 2014;23(1):120-128.

26. Panagopoulos J, Hancock MJ, Kongsted A, Hush J, Kent P. Does anterior trunk pain predict a different course of recovery in chronic low back pain? Pain. 2014;155(5):977-982.

27. Pinto RZ, Ferreira PH, Kongsted A, Ferreira ML, Maher CG, Kent P. Self-reported moderate-to-vigorous leisure time physical activity predicts less pain and disability over 12 months in chronic and persistent low back pain. Eur J Pain. 2014;18(8):1190-1198.

28. Dascanio V, Birks Y, Clark L, Fairhurst C, MacPherson H, Torgerson DJ. Randomized cohort trial was shown to be feasible for evaluating treatments in low back pain. J Clin Epidemiol. 2014;67(8): 940-946.

29. Neukamp M, Perler G, Pigott T, Munting E, Aebi M, Röder C. Spine Tango annual report 2012. Eur Spine J. 2013;22 Suppl 5:767-786.

30. Roovers JP. Registries: what level of evidence do they provide? Int Urogynecol J Pelvic Floor Dysfunct. 2007;18(10):1119-1120. 


\section{Publish your work in this journal}

Clinical Epidemiology is an international, peer-reviewed, open access, online journal focusing on disease and drug epidemiology, identification of risk factors and screening procedures to develop optimal preventative initiatives and programs. Specific topics include: diagnosis, prognosis, treatment, screening, prevention, risk factor modification,

Submit your manuscript here: http://www.dovepress.com/clinical-epidemiology-journal systematic reviews, risk \& safety of medical interventions, epidemiology \& biostatistical methods, and evaluation of guidelines, translational medicine, health policies \& economic evaluations. The manuscript management system is completely online and includes a very quick and fair peer-review system, which is all easy to use. 